\title{
Role of spin diffusion in current-induced domain wall motion for disordered ferromagnets
}

\author{
Collins Ashu Akosa, ${ }^{1}$ Won-Seok Kim, ${ }^{2}$ André Bisig,, 3 Mathias Kläui, ${ }^{3}$ Kyung-Jin Lee,,${ }^{2,5}$ and Aurélien Manchon ${ }^{1, *}$ \\ ${ }^{1}$ Materials Science and Engineering, Division of Physical Science and Engineering, KAUST, Thuwal 23955, Saudi Arabia \\ ${ }^{2}$ Department of Materials Science and Engineering, Korea University, Seoul 136-713, Korea \\ ${ }^{3}$ Institut für Physik, Johannes Gutenberg Universität Mainz, 55128 Mainz, Germany \\ ${ }^{4}$ IBM Research-Zurich, 8803 Rüschlikon, Switzerland \\ ${ }^{5}$ KU-KIST Graduate School of Converging Science and Technology, Korea University, Seoul 136-713, Korea \\ (Received 9 December 2014; revised manuscript received 19 February 2015; published 12 March 2015)
}

\begin{abstract}
Current-induced spin transfer torque and magnetization dynamics in the presence of spin diffusion in disordered magnetic textures is studied theoretically. We demonstrate using tight-binding calculations that weak, spinconserving impurity scattering dramatically enhances the nonadiabaticity. To further explore this mechanism, a phenomenological drift-diffusion model for incoherent spin transport is investigated. We show that incoherent spin diffusion indeed produces an additional spatially dependent torque of the form $\sim \nabla^{2}[\mathbf{m} \times(\mathbf{u} \cdot \nabla) \mathbf{m}]+$ $\xi \nabla^{2}[(\mathbf{u} \cdot \nabla) \mathbf{m}]$, where $\mathbf{m}$ is the local magnetization direction, $\mathbf{u}$ is the direction of injected current, and $\xi$ is a parameter characterizing the spin dynamics (precession, dephasing, and spin-flip). This torque, which scales as the inverse square of the domain wall width, only weakly enhances the longitudinal velocity of a transverse domain wall but significantly enhances the transverse velocity of vortex walls. The spatial-dependent spin transfer torque uncovered in this study is expected to have significant impact on the current-driven motion of abrupt two-dimensional textures such as vortices, skyrmions, and merons.
\end{abstract}

DOI: 10.1103/PhysRevB.91.094411

PACS number(s): 72.25.-b, 75.60.Ch

\section{INTRODUCTION}

The control of the magnetic state of nanoscale heterostructures [1] such as magnetic domain walls [2] and vortex cores [3] by a spin-polarized charge current is attracting increasing interest as a promising mechanism for innovative memory devices [4]. Identifying the nature of the torque exerted by the injected current on the domain wall itself has constituted a stimulating challenge resulting in the observation of unique dynamical behaviors [2-6] and raising seminal questions concerning the transport of itinerant spins in inhomogeneous magnetic textures [7-13]. The most widely accepted form of the spin transfer torque exerted by a charge current on a smoothly varying magnetic texture $\mathbf{m}(\mathbf{r}, t)$ is $[7,8]$

$$
\mathbf{T}=b_{J}(\mathbf{u} \cdot \nabla) \mathbf{m}-\beta b_{J} \mathbf{m} \times(\mathbf{u} \cdot \nabla) \mathbf{m},
$$

where $b_{J}$ is the adiabatic spin torque, $\beta$ describes the so-called nonadiabaticity of the spin torque and $\mathbf{u}$ is the direction of current injection. The nonadiabaticity $\beta$ is generated by different mechanisms such as spin relaxation $[7,10]$, spin-orbit coupling [14], and magnetic texture-induced spin mistracking [9-11]. In addition to nonadiabaticity, it has been found that the Gilbert damping $\alpha$ can also be affected (enhanced) by the spin texture [13]. From the viewpoint of domain wall dynamics, the longitudinal (in the direction of current injection) velocity for transverse walls and the transverse (perpendicular to the direction of current injection) velocity for vortex walls is controlled by the ratio of $\beta$ to $\alpha$ [8]. Therefore, experimental efforts have been expended in accurately determining $\beta$ and $\alpha$ for a wide range of magnetic materials and domain wall widths [2-6]. Experiments have shown that this ratio depends on the domain wall structure (Bloch, Néel, or vortex) and that vortex domain walls exhibit a much larger nonadiabaticity $(\beta \approx 8 \alpha$ to

\footnotetext{
*aurelien.manchon@kaust.edu.sa
}

10 $\alpha)$ [6,15-17] compared to transverse domain walls $(\beta \approx \alpha)$ as summarized in Table I. The authors attribute these large nonadiabaticities to the narrow character of domain walls in vortex structures $(\approx 10 \mathrm{~nm})$. As a matter of fact, Tatara et al. [9], Xiao et al. [11], and Wessely et al. [18] demonstrated that in abrupt domain walls the itinerant spin cannot adiabatically follow the local spin texture, resulting in an enhancement of the nonadiabaticity. Burrowes et al. [19] on the other hand, tested a very sharp transverse wall of about $1 \mathrm{~nm}$ using FePt nanowires and found that such a narrow domain wall does not cause a significant increase in the nonadiabaticity.

Theoretical investigations [10,23] have also shown a damped oscillatory behavior of the nonadiabatic torque when increasing the domain wall width, which may account for the nonadiabaticity enhancement for abrupt domain walls. However, these models are applied to transverse walls only and do not readily explain the observed differences between transverse and vortex walls.

The experimental observations $[6,15,16,19]$ indicate that the nature of the nonadiabatic spin torque exerted on abrupt magnetization patterns could be related to their dimensionality. Indeed, whereas a magnetic transverse wall varies along one direction only $\left(\partial_{x} \mathbf{m} \neq 0, \partial_{y} \mathbf{m}=0\right)$, the magnetization in a vortex structure varies along two directions $\left(\partial_{x} \mathbf{m} \neq\right.$ $0, \partial_{y} \mathbf{m} \neq 0$ ). Therefore, one approach to explain the different nonadiabaticities of abrupt transverse domain walls and vortex structures is to consider a mechanism that couples both $x$ and $y$ directions. It has been shown that a transverse spin current caused by anomalous Hall effect increases the transverse velocity of vortex cores while leaving the transverse domain walls essentially unchanged [24]. However, its contribution is of the order of the damping constant $\alpha$ and thus is insufficient to explain the results observed in Refs. $[6,15,16]$. An alternative mechanism is the diffusion of spin accumulation along the domain wall. This effect, neglected in the original theory of Zhang and $\mathrm{Li}$ [7], has recently been proposed to be responsible 
TABLE I. Summary of measurements of nonadiabaticity for different materials. The table entries show values of $\beta(\beta / \alpha)$ for vortex walls (VW), in-plane transverse walls (TW), and out-of-plane Bloch walls (BW).

\begin{tabular}{lc}
\hline \hline Material & $\beta(\beta / \alpha)$ \\
\hline Py (VW) & $0.018(2)[3], 0.04(8)[6]$ \\
& $0.073(9)[15], 0.15(10)[16], 0.15(9.2)[17]$ \\
Py (TW) & $0.01(1.2)[15], 0.02(1)[20], 0.13(10)[21]$ \\
FePt (BW) & $0.06(1)[19]$ \\
CoNi (BW) & $0.022(1)[19]$ \\
Pt/Co (BW) & $0.35(2)[22]$ \\
\hline \hline
\end{tabular}

for an enhancement of the nonadiabaticity of sharp domain walls [25].

In this article, we demonstrate both numerically and analytically that scattering against spin-independent disorder dramatically increases the nonadiabatic spin torque and can significantly affect the motion of abrupt domain walls. The physics of nonadiabaticity in magnetic domain walls involves two types of mechanisms: (i) the coherent precession of the spin around the local magnetization and (ii) spin dephasing and relaxation induced by disorder. In the absence of disorder, the spin precession around a smoothly varying magnetization results in a vanishingly small nonadiabaticity [11]. When a small amount of spin-independent disorder is introduced, the nonadiabaticity increases due to the enhancement of the spin dephasing, which we interpret in terms of an effective ElliottYafet spin-flip scattering enabled by the abrupt magnetic texture. As a consequence, the resulting nonadiabaticity increases when the domain wall width decreases and when increasing the strength and the amount of disorder. These features are demonstrated in Sec. II A using a tight-binding model (weak disorder regime) and Sec. II B using a drift-diffusion model (strong disorder regime). In Sec. III, we show that the resulting nonadiabatic torque significantly affects the current-driven motion of Bloch walls and vortex cores and may result in an effective increase of the transverse velocity of vortex walls by an order of magnitude. A discussion of the relevance of our study to previous experimental observations is given in Sec. IV, and Sec. V concludes this article.

\section{THEORETICAL MODEL}

\section{A. Tight-binding model for disordered ferromagnets}

In this section, we present a framework to calculate the local spin transfer torque for both ballistic and diffusive transport in magnetic textures. In particular, we consider a single-band tight-binding model on a two-dimensional square lattice as implemented in KWANT software [26,27]. Our system can be described by the Hamiltonian

$$
\mathcal{H}=\sum_{i} \epsilon_{i} \hat{c}_{i}^{+} \hat{c}_{i}-t \sum_{\langle i j\rangle} \hat{c}_{i}^{+} \hat{c}_{j}-\Delta_{\mathrm{ex}} \sum_{i} \hat{c}_{i}^{+} \mathbf{m}_{i} \cdot \hat{\boldsymbol{\sigma}} \hat{c}_{i},
$$

where $\epsilon_{i}$ is the onsite energy, $t$ is the hopping parameter, the sum $\langle i j\rangle$ is over nearest neighbors, $\mathbf{m}_{i}$ is a unit vector in the direction of the local moment at site $i, \Delta_{\mathrm{ex}}$ is the strength of the exchange energy that couples the local moment to the

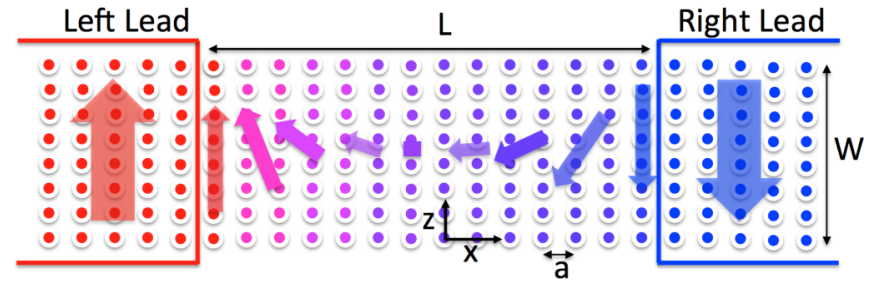

FIG. 1. (Color online) Sketch of the one-dimensional Bloch wall implemented on a two-dimensional square lattice as used in the tightbinding model described in the main text.

itinerant electrons with spin represented by the Pauli matrices $\hat{\boldsymbol{\sigma}} . \hat{c}_{i}^{+}=\left(c_{i}^{\uparrow}, c_{i}^{\downarrow}\right)^{+}$is the spinor form of the usual fermionic creation operator. Without loss of generality, we consider a charge current flowing along the $x$ axis and choose the $z$ axis as the quantization axis. Our calculations are performed on a onedimensional Bloch wall with spin texture as illustrated in Fig. 1 in spherical coordinates given by $\theta(x)=2 \tan ^{-1}(\exp [x / \Delta])$ and $\phi=\pi / 2$, where $\Delta$ is the domain wall width.

To calculate the transport properties of interest in our system, we first calculate the nonequilibrium spin density $\delta \mathbf{s}_{i}$ from the local spin density $\mathbf{s}_{i}$ for both electrons coming from the left $(\mathcal{L})$ and $\operatorname{right}(\mathcal{R})$, as $\delta \mathbf{s}_{i}=\mathbf{s}_{i}^{\mathcal{L}}-\mathbf{s}_{i}^{\mathcal{R}}$ [28]. The local spin torque $\mathbf{T}_{i}$ is defined as the torque exerted by the nonequilibrium spin density on the local moment at site $i$ and reads

$$
\mathbf{T}_{i}=\Delta_{\mathrm{ex}} \delta \mathbf{s}_{i} \times \mathbf{m}_{i} .
$$

To extract the different torque components, the torque is rewritten in the form $\mathbf{T}_{i}=T_{\text {ad }}^{i} \nabla_{x} \mathbf{m}_{i}+T_{\text {nad }}^{i} \mathbf{m}_{i} \times \nabla_{x} \mathbf{m}_{i}$ where $T_{\text {ad(nad) }}^{i}$ is the local adiabatic (nonadiabatic) torque component, from which the local nonadiabaticity parameter (defined as the ratio of the local nonadiabatic and adiabatic torques, i.e., $\beta_{i}=T_{\text {nad }}^{i} / T_{\text {ad }}^{i}$ ) is extracted. In all our calculations, we use the parameters: system size $401 \times 31 a^{2}$, where $a$ is the lattice constant, $t=1.0 \mathrm{eV}, \Delta_{\mathrm{ex}}=0.8 \mathrm{eV}$, and transport energy $E_{\mathrm{F}}=7.0 \mathrm{eV}$.

Figure 2 shows ballistic calculations of the spatial distribution of the adiabatic and nonadiabatic spin transfer torques for different current spin polarizations of the ferromagnet for a domain wall width of $8 a$. In our calculations, we tune the current spin polarization $P=\left(G^{\uparrow}-G^{\downarrow}\right) /\left(G^{\uparrow}+G^{\downarrow}\right), G^{\uparrow(\downarrow)}$ being the conductance for spin-up (spin-down) channel at the Fermi level, by shifting the onsite energy of the system. The nonadiabatic torque, which strongly depends on the current spin polarization, has been normalized by the nonadiabaticity at the center of the wall $\left(\beta_{\max }\right)$ and shows an oscillating behavior, which we attribute to the precession of the propagating states around the local moments in the form $e^{i\left(k^{\uparrow}-k^{\downarrow}\right) \cdot x}$, where $k^{\uparrow(\downarrow)}$ is the wave vector in the transport direction [34]. As shown in Fig. 3(a), the average nonadiabaticity defined as the average over space of the local nonadiabaticity, $\beta=\sum_{i} \beta_{i} / L$ with $L$ being the length of the system, is related to the domain wall width by $\beta=\beta_{0} \exp \left(-\Delta / \lambda_{L}\right)$, where $\lambda_{L}$ depends on the current spin polarization and controls the length scale for which the adiabatic approximation holds [11].

We model spin-conserving impurity scattering through the addition of a random potential $V_{i}$ to the constant onsite energy $\epsilon_{0}$ as $\epsilon_{i}=\epsilon_{0}+V_{i}$ such that $V_{i} \in\left[-0.5 V_{\text {imp }}, 0.5 V_{\text {imp }}\right]$. To 


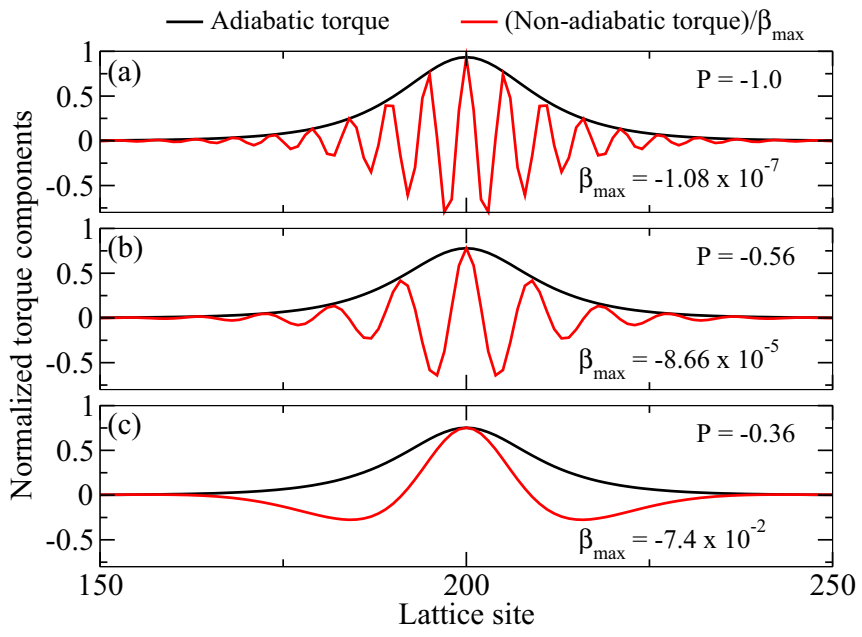

FIG. 2. (Color online) Spatial distribution of the adiabatic and nonadiabatic torque for different current spin polarization. (a) $P=$ -1.0 , (b) $P=-0.56$, and (c) $P=-0.36$. The adiabatic torque remains essentially constant, while the nonadiabatic torque shows strong depends on the current spin polarization.

calculate the transport properties of our system, we calculate the statistical average over a thousand different disorder configurations. For the range of impurities considered, the meanfree path is much larger than the domain wall width, which corresponds to realistic experimental situations. Figures 3(a) and 3(b) show that in the presence of spin-conserving impurity scattering, the nonadiabaticity is significantly increased as compared to the ballistic case and follows the empirical law $\tilde{\beta}=\tilde{\beta}_{0} \exp \left(-\Delta / \tilde{\lambda}_{L}\left[1-\Delta / \lambda_{L}^{*}\right]\right)$, where $\lambda_{L}^{*}$ and $\tilde{\lambda}_{L}$ depend on both the impurity strength and current spin polarization.

This result shows that (i) spin mistracking is the dominant mechanism in disorder-free systems and is only significant in weak ferromagnets and abrupt spin textures, which is consistent with Xiao et al. [11]. (ii) In the presence of

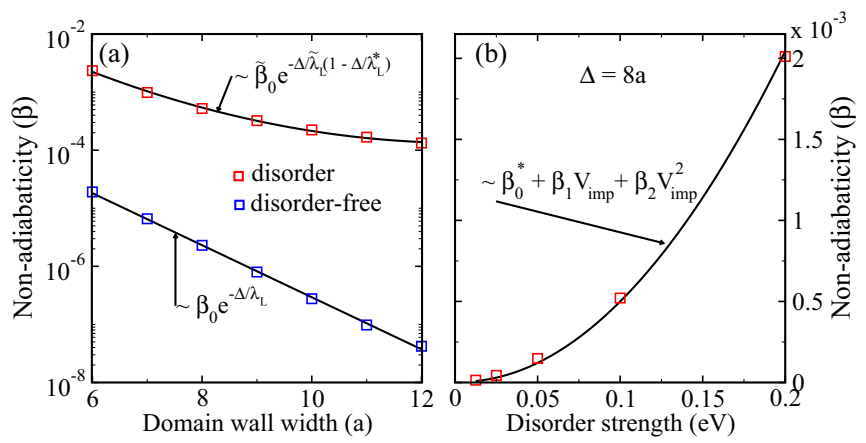

FIG. 3. (Color online) (a) Nonadiabaticity parameter as a function of the domain wall width in the absence (red) and presence of spin-conserving impurity scattering (black) of strength $V_{\text {imp }}=0.1 \mathrm{eV}$; (b) Non-adiabaticity parameter as a function of spin-conserving impurity scattering strength for a domain wall width of $8 \mathrm{a}$. In these calculations, we set the polarization to $P=-0.56$. In the absence of disorder, the nonadiabaticity is governed by spin mistracking while it is dominated by dephasing when introducing disorder in the structure. The fitted parameters are $\tilde{\beta}_{0}=2.92, \beta_{0}=8.9 \times 10^{-3}$, $\beta_{0}^{*}=4.27 \times 10^{-6}, \beta_{1}=2.87 \times 10^{-4}, \beta_{2}=5.24 \times 10^{-2}, \lambda_{L}=0.97$, $\tilde{\lambda}_{L}=0.64$, and $\lambda_{L}^{*}=25$. spin-conserving impurity scattering, the nonadiabaticity is enhanced by two to three orders of magnitude even in the absence of explicit spin-flip scattering. Such an enhancement can be attributed to the onset of spin relaxation due to the combined effect of momentum scattering and magnetic textures. Indeed, a magnetic texture induces a mixing of the spin states in the rotating frame of the magnetization. This spin mixing is, for instance, the physical ground of ballistic domain wall resistance [29]. In other words, a magnetic texture mixes the spin projections as spin-orbit coupling does. The introduction of spin-conserving disorder relaxes the linear momentum, and results in an Elliott-Yafet-type spin relaxation [30]. Therefore, disorder-induced momentum relaxation coupled with domain-wall-induced spin mixing results in the onset of spin relaxation. Since this spin relaxation has been recently shown to enhance the domain wall resistance [31], it is reasonable that it also induces an enhancement of the nonadiabaticity [see Ref. [9], Eq. (10)]. This demonstrates the seminal role played by disorder and momentum scattering on spin transfer torque in abrupt magnetic textures.

To further explore this mechanism, we address the nature of the nonadiabaticity in the diffusive regime in which disorder is treated incoherently. In this regime, the amount and strength of the disorder must be such that the mean-free path is smaller than the system size. However, modeling such a system using a quantum mechanical tight-binding model requires a large system size as well as averaging over a large number of disorder configurations and is therefore computationally demanding. Therefore, the nonadiabaticity in this regime is modeled following a semiclassical drift-diffusion approach.

\section{B. Drift-diffusion model}

We now investigate the role of spin diffusion within a driftdiffusion model, which in principle applies in the limit of strong disorder and strictly speaking is valid for domain wall widths larger than the electron mean-free path. The itinerant electrons evolving in magnetic textures are described by the one-electron Hamiltonian

$$
\hat{\mathcal{H}}=\frac{\hat{\mathbf{p}}^{2}}{2 m}+\Delta_{\mathrm{ex}} \hat{\boldsymbol{\sigma}} \cdot \mathbf{m}(\mathbf{r}, t),
$$

where $\mathbf{m}=\cos \phi \sin \theta \mathbf{e}_{x}+\sin \phi \sin \theta \mathbf{e}_{y}+\cos \theta \mathbf{e}_{z}$ is the timeand position-dependent magnetization direction $(|\mathbf{m}|=1), \hat{\mathbf{p}}$ is the momentum, $\hat{\sigma}$ is the Pauli matrix, and $\Delta_{\mathrm{ex}}$ is the exchange coupling energy. The itinerant electron spin operator satisfies the generalized spin continuity equation

$$
\partial_{t} \hat{\sigma}+\nabla \cdot \hat{\mathcal{J}}=\frac{1}{i \hbar}[\hat{\sigma}, \mathcal{H}]-\hat{\Gamma}_{\mathrm{re}}(\hat{\sigma}, \mathbf{m}),
$$

where $\hat{\mathcal{J}}$ is the spin current operator, $\hat{\Gamma}_{\text {re }}$ represents spin relaxation and dephasing due to scattering against spin-orbitcoupled, magnetic, and nonmagnetic impurities. The driftdiffusion equation for spin dynamics has been derived using quantum kinetics [32] and generalized random matrix theory [33] from which the spin accumulation $\mathbf{s}=\langle\hat{\sigma}\rangle$ in the diffusive regime satisfies the equation

$$
\partial_{t} \mathbf{S}=-\nabla \cdot \mathcal{J}-\frac{1}{\tau_{\mathrm{ex}}} \mathbf{S} \times \mathbf{m}-\Gamma_{\mathrm{re}}(\mathbf{s}, \mathbf{m}),
$$


where $\tau_{\mathrm{ex}}=\hbar / 2 \Delta_{\mathrm{ex}}$ is the spin precession time $\left(\approx 10^{-15} \mathrm{~s}\right.$ to $\left.10^{-14} \mathrm{~s}\right), \mathcal{J}=\langle\hat{\mathcal{J}}\rangle$ is the spin current density tensor such that $\mathcal{J}=-\mathcal{D} \nabla \otimes \mathbf{s}$, where $\mathcal{D}$ is the diffusion constant and $\Gamma_{\text {re }}=$ $\left\langle\hat{\Gamma}_{\text {re }}\right\rangle$. In systems such as disordered ferromagnetic metallic nanowires, the local momentum scattering tends to counteract the influence of the external electric field resulting in an additional diffusion term in both charge and spin currents [33].

The dynamics of the magnetization and that of the itinerant spins are considered to be decoupled since the dynamics of conduction electrons is fast compared to that of magnetization. As a result, the spin density can be written in the form $\mathbf{s}=$ $n_{s} \mathbf{m}+\delta \mathbf{s}$, where $n_{s}(\delta \mathbf{s})$ is the (nonequilibrium) equilibrium spin density $\left(n_{s} \approx 10^{-2} M_{s}\right)$. Similarly, the spin current density takes the form $\mathcal{J}=\mathcal{J}_{0}+\delta \mathcal{J}$. $\mathcal{J}_{0}=-b_{J} \mathbf{u} \otimes \mathbf{m}$ is the adiabatic spin current density, where $b_{J}=\mu_{B} P G_{0} E / e, P$ is the spin polarization, $\mu_{B}$ is the Bohr magneton, $G_{0}$ is the electrical conductivity in the absence of magnetic texture, $\mathbf{u}$ is the direction of injected current. $\delta \mathcal{J}=-\mathcal{D} \nabla \delta \mathbf{s}$ is the nonadiabatic spin current density, which arises from carrier diffusion. Using the relaxation time approximation, $\Gamma_{\mathrm{re}}$ is given by

$$
\Gamma_{\mathrm{re}}(\mathbf{s}, \mathbf{m})=\frac{1}{\tau_{\mathrm{sf}}} \delta \mathbf{s}+\frac{1}{\tau_{\phi}} \mathbf{m} \times(\delta \mathbf{s} \times \mathbf{m}),
$$

where $\tau_{\mathrm{sf}}$ is the phenomenological spin-flip relaxation time $\left(\approx 10^{-13}\right.$ s to $\left.10^{-12} \mathrm{~s}\right)$ and $\tau_{\phi}$ is the spin dephasing time.

Note that Eq. (7) differs from Ref. [7] by the presence of a dephasing term $\left(\propto 1 / \tau_{\phi}\right)$, which arises from the destructive interference of nonequilibrium precessing spins with different wave vector directions. Microscopic investigations of spin dephasing at the interface between a normal metal and a strong ferromagnet using realistic Fermi surfaces have shown that this effect destroys the transverse component of itinerant spins within a few monolayers [34]. In bulk disordered ferromagnets, recent derivations using quantum Boltzmann equation [35] or generalized random matrix theory [36] have demonstrated that the interplay between spin precession and impurity scattering introduces a spin dephasing. The presence of spin dephasing, has an important implication in terms of spin torque definition. Indeed, the torque $\mathbf{T}$ is defined as the amount of spin current absorbed by the local magnetization less the spin density lost to the environment. Therefore from Eq. (6), we obtain

$$
\mathbf{T}=-\nabla \cdot \mathcal{J}-\frac{1}{\tau_{\mathrm{sf}}} \delta \mathbf{s}=\frac{1}{\tau_{\mathrm{ex}}} \delta \mathbf{s} \times \mathbf{m}+\frac{1}{\tau_{\phi}} \mathbf{m} \times(\delta \mathbf{s} \times \mathbf{m}) .
$$

In the following, we use this definition to express the spin torque up to the third order in spatial gradient of the magnetization.

\section{Spin transfer torque from drift diffusion}

In the framework of the above representation, the drift-diffusion equation for the nonequilibrium spin density is given by

$$
\begin{aligned}
& -\mathcal{D} \nabla^{2} \delta \mathbf{s}+\frac{1}{\tau_{\mathrm{ex}}} \delta \mathbf{s} \times \mathbf{m}+\frac{1}{\tau_{\phi}} \mathbf{m} \times(\delta \mathbf{s} \times \mathbf{m})+\frac{1}{\tau_{\mathrm{sf}}} \delta \mathbf{s} \\
& =b_{J} \nabla_{x} \mathbf{m}-\partial_{t} \delta \mathbf{s}-n_{s} \partial_{t} \mathbf{m}+\mathcal{D} n_{s} \nabla^{2} \mathbf{m} .
\end{aligned}
$$

The right-hand side (RHS) of Eq. (9) acts as a source of itinerant spin dynamics within the spin texture for current applied along the $x$ direction. We solve Eq. (9) for the steadystate $\left(\partial_{t} \delta \mathbf{s}=\partial_{t} \mathbf{m}=0\right)$ solution of the nonequilibrium spin density from which the torque can be calculated using Eq. (8).

In systems with wide and smoothly varying spin textures such that the domain wall width (radius of vortex core) $W$ and the spin diffusion length $\lambda_{\mathrm{sf}}=\sqrt{\mathcal{D} \tau_{\mathrm{sf}}}$ satisfies $W^{2} \gg \lambda_{\mathrm{sf}}^{2}$, the first term in the left-hand side (LHS) of Eq. (9) can be neglected and in this case, the itinerant nonequilibrium spin density reduces to $[7,8]$

$$
\delta \mathbf{s} \approx b_{J} \tilde{\tau}_{\mathrm{ex}}\left[\xi \nabla_{x} \mathbf{m}+\mathbf{m} \times \nabla_{x} \mathbf{m}\right],
$$

where $\xi=\beta_{0}+\chi, \quad \beta_{0}=\tau_{\mathrm{ex}} / \tau_{\mathrm{sf}}, \quad \chi=\tau_{\mathrm{ex}} / \tau_{\phi}$ and $\tilde{\tau}_{\mathrm{ex}}=$ $\tau_{\text {ex }} /\left(1+\xi^{2}\right)$.

However, for abrupt spin textures or vortex structure for which $W^{2} \sim \lambda_{\text {sf }}^{2}$, the first term in the LHS of Eq. (9) can no longer be neglected. Before we present the complete analytical results, we first attempt to give some insight of the impact of the diffusion term [first term in LHS of Eq. (9)] on the spin torque.

Let us start with a more general consideration of vector representation in space. Since the unit vectors in the direction of $\left(\mathbf{m}, \nabla_{x} \mathbf{m}, \mathbf{m} \times \nabla_{x} \mathbf{m}\right)$ form a basis in three dimensions, any vector in space can be written as linear combination of these basis vectors. Therefore, without loss of generality, we can write the first term in the LHS of Eq. (9) in the form [37]

$$
-\mathcal{D} \nabla^{2} \delta \mathbf{s}=\frac{\mathcal{D}}{W^{2}}\left[\mathcal{G} \delta \mathbf{s}+\mathcal{B}_{a} \nabla_{x} \mathbf{m}+\mathcal{B}_{n} \mathbf{m} \times \nabla_{x} \mathbf{m}\right],
$$

where $\mathcal{G}, \mathcal{B}_{a}$ and $\mathcal{B}_{n}$ are spatially and spin-texture-dependent scalars. Inserting Eq. (11) into Eq. (9) we obtain

$$
\begin{gathered}
\frac{1}{\tau_{\mathrm{ex}}} \delta \mathbf{s} \times \mathbf{m}+\frac{1}{\tau_{\phi}} \mathbf{m} \times(\delta \mathbf{s} \times \mathbf{m})+\frac{1}{\tau_{\mathrm{sf}}}\left(1+\mathcal{G} \frac{\lambda_{\mathrm{sf}}^{2}}{W^{2}}\right) \delta \mathbf{s} \\
=\left(b_{J}-\mathcal{B}_{a} \frac{\mathcal{D}}{W^{2}}\right) \nabla_{x} \mathbf{m}-\mathcal{B}_{n} \frac{\mathcal{D}}{W^{2}} \mathbf{m} \times \nabla_{x} \mathbf{m} .
\end{gathered}
$$

We have made no assumption in arriving at Eq. (12), which is a general steady-state solution of Eq. (9). Moreover, its form depicts a very simple but instructive aspect of abrupt spin textures compared to wide and smoothly varying textures or homogeneous ferromagnets.

First, in the LHS of Eq. (12), the third term represents a spatially dependent renormalization of the spin diffusion length $\lambda_{\text {eff }}$ given by

$$
\frac{1}{\lambda_{\mathrm{eff}}^{2}}=\frac{1}{\lambda_{\mathrm{sf}}^{2}}\left(1+\mathcal{G}(\mathbf{r}) \frac{\lambda_{\mathrm{sf}}^{2}}{W^{2}}\right) .
$$

This renormalization, which is due to spin diffusion, is as expected strongest at the domain wall center and reduces outwardly as represented by the coefficient $\mathcal{G}$ as in the case of Bloch wall and vortex structure (see Sec. III).

Second, the renormalization of the spin diffusion length (the effective spin diffusion length is reduced within the domain wall for abrupt textures), results in an effective enhancement of the nonadiabaticity of the texture represented at first-order approximation as

$$
\beta_{\mathrm{eff}}=\beta_{0}\left(1+\mathcal{G}(\mathbf{r}) \frac{\lambda_{\mathrm{sf}}^{2}}{W^{2}}\right)
$$


The complete picture of the effect of spin diffusion can be obtained after solving for the nonequilibrium spin density from Eq. (12) and using Eq. (8) to obtain the torque. Without going into much details of the analytics, the spin torque is given by

$$
\mathbf{T}=\left(\tilde{\eta} b_{J}-\eta_{\text {diff }}\right) \nabla_{x} \mathbf{m}-\left(\tilde{\beta} b_{J}+\beta_{\text {diff }}\right) \mathbf{m} \times \nabla_{x} \mathbf{m},
$$

where $\tilde{\eta}=(1+\chi \xi) /\left(1+\xi^{2}\right)$ and $\tilde{\beta}=\beta_{0} /\left(1+\xi^{2}\right)$ are the adiabatic and nonadiabatic torque in the absence of spin diffusion respectively [this represents the spin torque obtained from the spin density given by Eq. (10)]. The contribution of spin diffusion to the adiabatic and nonadiabatic torques are $\eta_{\text {diff }}$ and $\beta_{\text {diff }}$ respectively given as

$$
\eta_{\text {diff }}=\frac{\mathcal{D}}{W^{2}}\left[\tilde{\eta} \mathcal{B}_{a}+\tilde{\beta} \mathcal{B}_{n}\right] \text { and } \beta_{\text {diff }}=\frac{\mathcal{D}}{W^{2}}\left[\tilde{\eta} \mathcal{B}_{n}-\tilde{\beta} \mathcal{B}_{a}\right]
$$

Notice that spin diffusion reduces (enhances) the adiabatic (nonadiabatic) torque, and hence enhances the nonadiabaticity parameter.

Therefore, it appears clear that the standard torque can be significantly modified by effects due to spin diffusion, which only becomes dominant over ballistic spin mistracking for abrupt spin textures or vortex structures. In particular, for long spin precession lengths (in the case of weak ferromagnets) or abrupt spin textures (strong anisotropy or vortex structure), there is an enhancement of nonadiabaticity as the spin dephasing is not strong enough to compensate the mistracking due to the spin precession.

In the following section we combine fully numerical and analytical treatment of the domain wall velocity for a Bloch wall and a vortex structure to uncover the importance of corrections due to spin diffusion. Our numerical treatment entails (i) solving Eq. (9) using COMSOL MULTIPHYSICS software, (ii) calculating the spin torque and its components using the torque definition in Eq. (8), (iii) calculating the velocities of the domain wall using Thiele's formalism for spin textures [39].

\section{CURRENT-INDUCED DOMAIN WALL MOTION}

We study the influence of the spin diffusion on two typical magnetization patterns: a Bloch wall and a vortex structure with magnetization profile as shown in Fig. 4. In both cases, the magnetization dynamics is governed by the Landau-LifshitzGilbert-Slonczewski (LLGS) equation

$$
\partial_{t} \mathbf{m}=-\gamma \mathbf{m} \times \mathbf{H}_{\mathrm{eff}}+\alpha \mathbf{m} \times \partial_{t} \mathbf{m}+\mathbf{T},
$$

where $\gamma$ is the gyromatic ratio, $\alpha$ is the Gilbert damping constant, $\mathbf{T}$ is the spin transfer torque. $\mathbf{H}_{\mathrm{eff}}$ is the effective field given by

$$
\begin{aligned}
\mathbf{H}_{\mathrm{eff}}= & 2 A \nabla^{2} \mathbf{m}+\left[H_{z}+\left(H_{k}+H_{d z}\right) \cos \theta\right] \mathbf{e}_{z} \\
& +H_{d x} \cos \phi \sin \phi \mathbf{e}_{x}+H_{d y} \sin \phi \sin \theta \mathbf{e}_{y},
\end{aligned}
$$

where $A$ is the exchange constant, $H_{z}$ is the external applied field along the $z$ axis, $H_{k}$ is the uniaxial anisotropy field, and $H_{d i}(i=x, y, z)$ are the demagnetizing field along the $i$ axis whose relative strength depends on the dimensions of the nanowire and satisfies the relation $\sum_{i} H_{d i}=-4 \pi M_{s}$, where $M_{s}$ is the saturation magnetization of the ferromagnet.
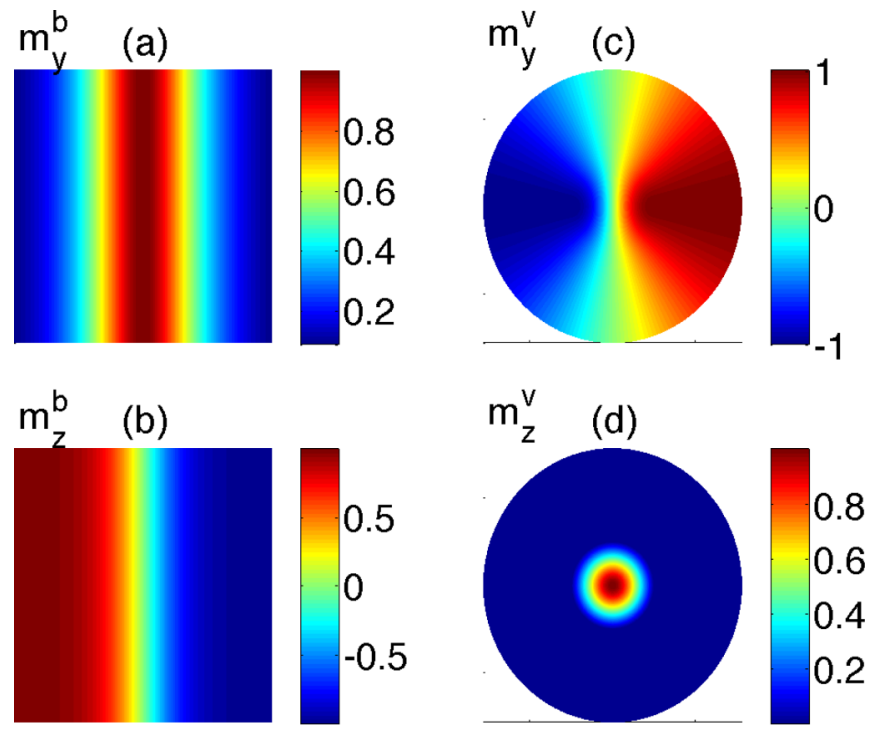

FIG. 4. (Color online) Spatial profile of the $y$ (a), (c) and $z$ components (b), (d) of the magnetization of a typical Bloch wall (a), (b) and vortex core (c), (d).

\section{A. Transverse wall}

We focus on the case of perpendicularly magnetized systems, as the case of in-plane magnetized systems produces similar results. In a perpendicularly magnetized nanowire, a one-dimensional Bloch wall is described by $\theta(x)=$ $2 \tan ^{-1}\left(e^{(x-X) / \Delta}\right), \phi=\phi(t)$, where $X$ and $\Delta$ are the wall center and width respectively. Figures 4(a) and 4(b) show the $y$ and $z$ components, respectively, of the magnetization of the wall. To calculate the geometric scalars $\mathcal{G}, \mathcal{B}_{a}, \mathcal{B}_{n}$, and $\mathcal{B}$, we proceed by gradient expansion method, which entails starting with the zeroth-order solution of Eq. (10) for $\delta \mathbf{s}$ to get

$$
-\mathcal{D} \nabla^{2} \delta \mathbf{s}=\frac{\mathcal{D}}{\Delta^{2}}\left[\xi\left(3 \sin ^{2} \theta-1\right) \nabla_{x} \mathbf{m}+\cos 2 \theta \mathbf{m} \times \nabla_{x} \mathbf{m}\right] .
$$

Next, Eq. (19) is rewritten in the form of Eq. (11) and comparing coefficients of the basis vectors with Eq. (11) we obtain $\mathcal{G}=\xi\left(3 \sin ^{2} \theta-1\right), \mathcal{B}_{a}=b_{J} \tilde{\tau}_{\mathrm{ex}}(1-\xi) \mathcal{G}, \mathcal{B}_{n}=$ $b_{J} \tilde{\tau}_{\mathrm{ex}}(\mathcal{G}+\cos 2 \theta)$ and $\mathcal{B}=0$.

The dynamics of the domain wall is given by the coupled differential equations

$$
\begin{aligned}
& \partial_{\tau} X=\frac{\Delta}{\sin \theta}\left[\alpha \gamma H_{\theta}+\gamma H_{\phi}+\mathbf{T} \cdot \mathbf{e}_{\theta}-\alpha \mathbf{T} \cdot \mathbf{e}_{\phi}\right], \\
& \partial_{\tau} \phi=\frac{1}{\sin \theta}\left[\alpha \gamma H_{\phi}-\gamma H_{\theta}+\mathbf{T} \cdot \mathbf{e}_{\phi}+\alpha \mathbf{T} \cdot \mathbf{e}_{\theta}\right],
\end{aligned}
$$

where $\tau=t /\left(1+\alpha^{2}\right), \quad \mathbf{e}_{\phi}=\cos \phi \mathbf{e}_{y}-\sin \phi \mathbf{e}_{x}, \quad \mathbf{e}_{\theta}=\cos$ $\phi \cos \theta \mathbf{e}_{x}+\sin \phi \cos \theta \mathbf{e}_{y}-\sin \theta \mathbf{e}_{z}, H_{\theta, \phi}=\mathbf{H}_{\mathrm{eff}} \cdot \mathbf{e}_{\theta, \phi}$, and $\mathbf{T}$ is the total spin transfer torque calculated from Eq. (15).

In the absence of external applied field, at the lowest order, we obtain the average velocity of the domain wall below the Walker breakdown $\left(\partial_{\tau} \phi=0\right)$ as

$$
v_{x}^{<} \approx-\frac{\tilde{\beta}}{\alpha}\left(1+\frac{1}{3} \beta^{*}[1-2 \xi] \frac{\lambda_{\mathrm{sf}}^{2}}{\Delta^{2}}\right) b_{J},
$$

where $\beta^{*}=\tilde{\eta}-\tilde{\beta} \xi$. 

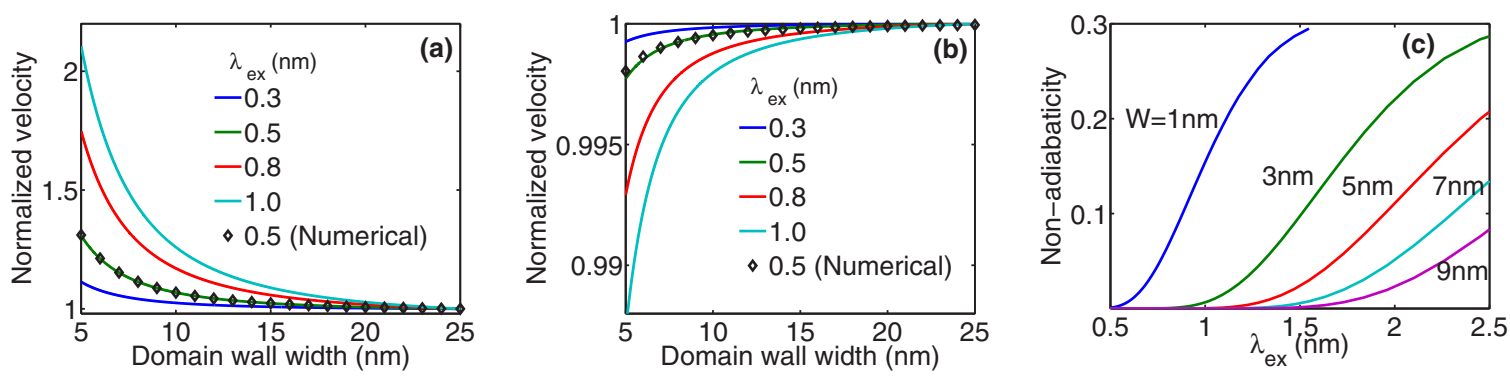

FIG. 5. (Color online) Normalized velocity of a one-dimensional Bloch wall, below $v_{x}^{<}$(a) and above Walker breakdown $v_{x}^{>}$(b). Numerical and analytic results are shown with dotted and solid lines respectively. The parameters used are $\alpha=0.005$ and $\beta_{0}=0.01, \chi=0.2$. (c) Spin-mistracking nonadiabaticity as a function of the transverse spin diffusion length $\lambda_{\text {ex }}$ for decreasing domain wall widths, calculated based on Ref. [11].

Above the Walker breakdown $\left(\partial_{\tau} \phi \neq 0\right)$, the average velocity of the domain wall is given by

$$
v_{x}^{>} \approx \frac{-b_{J}}{1+\alpha^{2}}\left(\tilde{\eta}+\alpha \tilde{\beta}-\frac{1}{3} \tilde{\beta}\left[\eta^{*}-\alpha \beta^{*}[1-2 \xi]+2 \xi \tilde{\eta}\right] \frac{\lambda_{\mathrm{sf}}^{2}}{\Delta^{2}}\right)
$$

where $\eta^{*}=\tilde{\eta} \xi+\tilde{\beta}$.

The additional nonadiabaticity given by the second term in the RHS of Eq. (22) is governed by the ratio $\lambda_{\text {sf }}^{2} / \Delta^{2}$, and since the velocity of the domain wall is governed by the nonadiabatic torque below the Walker breakdown, spin diffusion only significantly affects the longitudinal wall velocity below the Walker breakdown by enhancing the effective nonadiabaticity [cf. Eq. (14)].

Figures 5(a) and 5(b) display analytical and numerical results of the velocities of a Bloch wall as a function of the domain wall width $\Delta$. The velocities are normalized to the case without spin diffusion (wide and smoothly varying texture). Below the Walker breakdown [Fig. 5(a)], the velocity is moderately affected by the domain wall width. For example, when $\Delta=5 \mathrm{~nm}$, the normalized velocity increases by about a factor of 2 for $\lambda_{\mathrm{ex}}=0.8 \mathrm{~nm}$ (equivalent to $\mathrm{NiFe}$ with $\Delta_{\mathrm{ex}} \approx$ $0.5 \mathrm{eV}$, see also Ref. [38]). Above the Walker breakdown [Fig. 5(b)], the velocity is simply not affected by the domain wall width (less than 1\%). As can be seen from the numerical calculations for $\lambda_{\mathrm{ex}}=0.5 \mathrm{~nm}$, they are in very good agreement with our analytical predictions.

To assess the importance of the spin diffusion mechanism compared to the ballistic spin mistracking mechanism, we numerically calculated the nonadiabaticity caused by this latter mechanism based on Ref. [11]. The nonadiabaticity parameter evaluated numerically is defined as the ratio between the nonadiabatic torque and the adiabatic torque at the center of the wall. From Fig. 5(c), we see that the estimated contribution of the ballistic spin mistracking to the nonadiabaticity remains very limited and since most of the ferromagnetic materials used in experiments are strong ferromagnets $\left(\lambda_{\mathrm{ex}}<0.8 \mathrm{~nm}\right)$, this torque has a sizable influence for abrupt domain walls only.

\section{B. Vortex structure}

In a two-dimensional magnetic stripe, a vortex structure can be described by $\theta(x, y)=2 \tan ^{-1}\left(r / r_{0}\right)$ for $r=\sqrt{x^{2}+y^{2}} \leqslant$ $r_{0}, \theta=\pi / 2$ for $r_{0}<r \leqslant R$, and $\phi=\operatorname{Arg}(x, y)+\pi / 2$, where
$r_{0}(R)$ is the inner (outer) radius of the vortex core. Figures 4(c) and 4(d) show the $y$ and $z$ components of the magnetization. As in the case for the Bloch wall, we calculate the geometric scalars $\mathcal{G}, \mathcal{B}_{a}$, and $\mathcal{B}_{n}$ by gradient expansion method to obtain

$$
-\mathcal{D} \nabla^{2} \delta \mathbf{s}=\frac{\mathcal{D}}{r_{0}^{2}} \frac{2 r_{0}^{2}}{r^{2}} \sin ^{2} \theta\left[\xi \nabla_{x} \mathbf{m}+\mathbf{m} \times \nabla_{x} \mathbf{m}\right],
$$

and the geometric scalars $\mathcal{G}=\frac{2 r_{0}^{2}}{r^{2}} \xi \sin ^{2} \theta, \mathcal{B}_{a}=b_{J} \tilde{\tau}_{\text {ex }}(1-$ $\xi) \mathcal{G}$ and $\mathcal{B}_{n}=b_{J} \tilde{\tau}_{\text {ex }}(1-\xi) \mathcal{G} / \xi$.

To extract the velocities, we use Thiele's description in which Eq. (17) can be expressed in the form of the sum of forces exerted on the wall [39]. By multiplying Eq. (17) on the left by $\mathbf{m} \times$ and projecting the obtained equation on $-\partial_{i} \mathbf{m}$ one obtains

$$
\begin{aligned}
& -\int_{\Omega}\left[\partial_{t} \mathbf{m} \cdot\left(\alpha \partial_{i} \mathbf{m}+\mathbf{m} \times \partial_{i} \mathbf{m}\right)\right] \\
& \quad=\int_{\Omega}\left[\partial_{i} \mathbf{m} \cdot \gamma \mathbf{H}_{\mathrm{eff}}+\mathbf{T} \cdot\left(\mathbf{m} \times \partial_{i} \mathbf{m}\right)\right],
\end{aligned}
$$

for $i=x, y$. The integral $\int_{\Omega} d \Omega$ runs over the volume $\Omega$ of the magnetic stripe.

In the absence of external applied field, after some algebra, the longitudinal and transverse velocities of the vortex core are given by

$$
\begin{gathered}
v_{x}=-\frac{b_{J}}{1+\alpha^{2}}\left[\tilde{\eta}+\alpha \tilde{\beta}-\mathcal{R} \tilde{\beta}\left(\eta^{*}-\alpha \beta^{*}\right) \frac{\lambda_{\mathrm{sf}}^{2}}{r_{0}^{2}}\right], \\
v_{y}=\frac{b_{J}}{1+\alpha^{2}}\left[\tilde{\beta}-\alpha \tilde{\eta}+\mathcal{R} \tilde{\beta}\left(\beta^{*}+\alpha \eta^{*}\right) \frac{\lambda_{\mathrm{sf}}^{2}}{r_{0}^{2}}\right],
\end{gathered}
$$

where $\mathcal{R}$ is a geometric factor given by $\mathcal{R}=[17 / 3-$ $\left.r_{0}^{2} / R^{2}\right] /\left[1+\ln \left(R / r_{0}\right)\right]$.

Figures 6(a) and 6(b) display the transverse and longitudinal velocities of the vortex core as a function of the core radius $r_{0}$. While the longitudinal velocity is not significantly affected, the transverse velocity is dramatically enhanced in the presence of spin diffusion. For $r_{0}=5 \mathrm{~nm}$, the transverse velocity can be increased by a factor of 10 for a transverse spin diffusion length $\lambda_{\mathrm{ex}}=0.8 \mathrm{~nm}$. This key result shows that spin diffusion cannot be neglected for vortex spin textures, in contrast to previous belief where only much more abrupt spin structures were considered to lead to an enhanced $\beta$. This indicates that the traditional way to extract $\beta$ must be reconsidered and that a more in-depth analysis of the velocities needs to be 

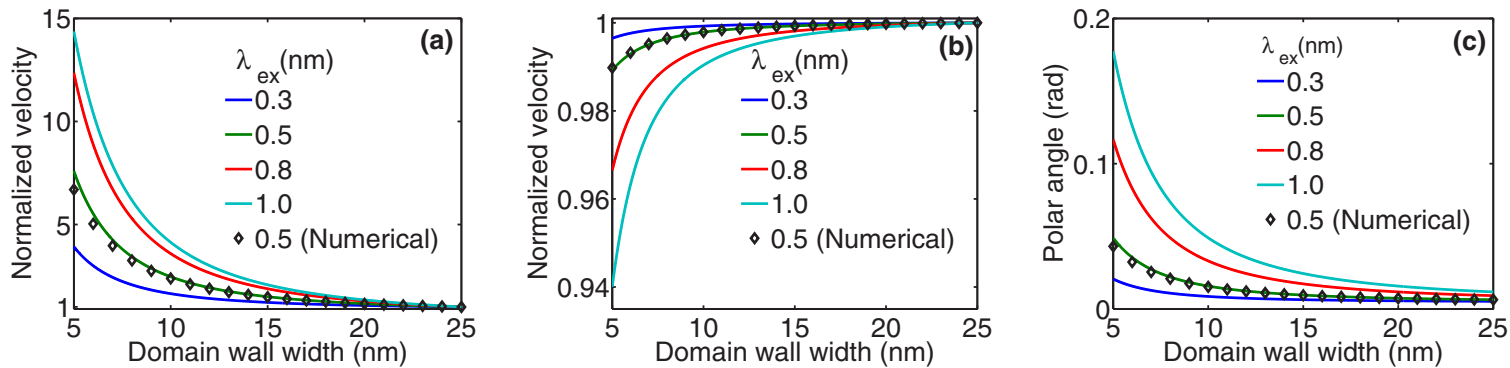

FIG. 6. (Color online) Normalized transverse $v_{y}$ (a) and longitudinal $v_{x}$ (b) velocities of the vortex core. (c) Polar angle of the vortex core as a function of the core radius. Numerical and analytic results are shown with dotted and solid lines respectively. The parameters used are $R=150 \mathrm{~nm}, \alpha=0.005, \beta_{0}=0.01$, and $\chi=0.2$.

performed. This can be phenomenologically understood as the renormalization of the effective spin diffusion length as itinerant electrons move across the vortex core represented by

$$
\lambda_{\text {eff }}=\lambda_{\mathrm{sf}} / \sqrt{1+\frac{8 \xi r_{0}^{4}}{\left(r^{2}+r_{0}^{2}\right)^{2}} \frac{\lambda_{\mathrm{sf}}^{2}}{r_{0}^{2}}} .
$$

This shows that as itinerant electrons flow across the vortex core, their effective spin diffusion length reduces by a factor of $\sqrt{1+8 \xi \lambda_{\text {sf }}^{2} / r_{0}^{2}}$ which represents an equivalent enhancement of the nonadiabaticity parameter. As can be seen from they for $\lambda_{\mathrm{ex}}=0.5 \mathrm{~nm}$, the numerical calculations are in very good agreement with our analytical predictions. It should be noted however that the analytical treatment does not include higher-order terms, which are taken into account in the numerical calculations, resulting in the slight deviation at very short core radius.

Another way to extract the nonadiabaticity parameter is to estimate the polar angle $\tan ^{-1}\left(v_{y} / v_{x}\right)$ acquired by the vortex core after current injection [16]. As shown in Fig. 6(c), the polar angle is dramatically enhanced at small core sizes due to the transverse spin diffusion. Therefore, for vortex cores, the transverse spin diffusion provides a sizable contribution to the nonadiabaticity.

\section{DISCUSSION}

Up to now, only spin-flip and ballistic mistracking were known to be responsible for the nonadiabatic torque. In Sec. II A, we show that in a disordered system even in the absence of explicit spin flip, spin dephasing induced by weak disorder dramatically enhances the nonadiabaticity parameter. This is interpreted in terms of an Elliott-Yafet-type relaxation where the magnetic texture plays the role of spin-orbit coupling. Increasing the amount of disorder (or reducing the width of the domain wall) results in an increase of the nonadiabaticity. In the case of strong disorder, the system is dragged towards the diffusive regime (i.e., the mean-free path becomes smaller than the system size). However, as mentioned in Sec. II A, modeling such a regime using a quantum mechanical tight-binding model is computationally demanding and we adopt a semiclassical drift-diffusion approach to explore this regime. Analytical expressions of the nonadiabaticity are derived in the diffusive limit and we explicitly show that the spin diffusion length is enhanced by the conjunction of spin diffusion and sharp magnetic texture [see Eq. (13)], resulting in a larger nonadiabaticity parameter [see Eq. (14)], which is consistent with the results from the tight-binding model. Our calculations [see Figs. 6(b) and 6(c)] are consistent with the large nonadiabaticities measured in Refs. $[6,15,16]$ for vortex cores in $\mathrm{NiFe}$. In these structures the transverse spin diffusion length is about $\lambda_{\mathrm{ex}} \approx 0.8 \mathrm{~nm}[34,38]$ and the radius $r_{0} \approx 5 \mathrm{~nm}$, which yields effective velocities about 10 times larger than in the absence of spin diffusion. Although the length scale for which our model lies within the borderline regime of validity of the drift-diffusion formalism, our calculations provide a consistent explanation of the enhancement of the nonadiabaticity parameter and hence domain wall velocity between the mistracking-dominated (clean) and spin-diffusion-dominated regimes (strong disorder). Finally, in magnetic wires with strong perpendicular magnetic anisotropy, such as $\mathrm{Co} / \mathrm{Ni}, \mathrm{FePt}$ or $\mathrm{Co} / \mathrm{Pt}[19,22]$, the domain wall width is quite small $(\sim 5 \mathrm{~nm})$ and therefore one would expect the nonadiabaticity to be quite large. Nonetheless, these materials are strong ferromagnets and therefore the mistracking between the itinerant spin and the magnetic texture is quite weak, resulting in a weak correction to the nonadiabaticity $(\beta \sim \alpha)$.

\section{CONCLUSION}

The role of diffusion of spin accumulation in currentinduced domain wall motion has been studied theoretically using both a tight-binding model and a drift-diffusion approach. In particular, we consider an abrupt Bloch wall and a vortex structure and investigate how spin diffusion affects the nonadiabaticity parameter and hence the velocity of the wall. Our results show that while spin diffusion only has a moderate effect on the longitudinal velocity of a Bloch wall, it significantly enhances the transverse velocity of the vortex core up to an order of magnitude. We show that for abrupt spin textures, the diffusion of spin accumulation produces a supplementary spatially dependent torque, which depends on the topology of the texture and is proportional to the square of the ratio of the effective spin diffusion length and the domain wall width. Our results uncover the significant difference between a transverse wall and a vortex core: in the latter, the abrupt texture results in a stronger renormalization (reduction) of the effective spin diffusion length resulting in a stronger enhancement of the nonadiabaticity parameter.

\section{ACKNOWLEDGMENTS}

The authors thank M. D. Stiles and X. Waintal for inspiring discussions. A.C.A. and A.M. were supported by the King 
Abdullah University of Science and Technology (KAUST). K.J.L. acknowledges financial support from NRF (Korea) (Grant No. 2013R1A2A2A01013188), and M.K. acknowledges the DFG and the EU (WALL, FP7-PEOPLE-2013-ITN
608031; MASPIC, ERC-2007-StG 208162) as well as the Center of Innovative and Emerging Materials at Johannes Gutenberg University Mainz and the Graduate School of Excellence Materials Science in Mainz (GSC 266).
[1] J. C. Slonczewski, J. Magn. Magn. Mater. 159, L1 (1996); L. Berger, Phys. Rev. B 54, 9353 (1996).

[2] M. Kläui, P.-O. Jubert, R. Allenspach, A. Bischof, J. A. C. Bland, G. Faini, U. Rüdiger, C. A. F. Vaz, L. Vila, and C. Vouille, Phys. Rev. Lett. 95, 026601 (2005); M. Hayashi, L. Thomas, C. Rettner, R. Moriya, Y. B. Bazaliy, and S. S. P. Parkin, ibid. 98, 037204 (2007); M. Yamanouchi, D. Chiba, F. Matsukura, and H. Ohno, Nature (London) 428, 539 (2004); A. Yamaguchi, T. Ono, S. Nasu, K. Miyake, K. Mibu, and T. Shinjo, Phys. Rev. Lett. 96, 179904(E) (2006).

[3] R. Moriya, L. Thomas, M. Hayashi, Y. B. Bazaliy, C. Rettner, and S. S. P. Parkin, Nature Phys. 4, 368 (2008); K. Yamada, S. Kasai, Y. Nakatani, K. Kobayashi, H. Kohno, A. Thiaville, and T. Ono, Nature Mater. 6, 270 (2007).

[4] S. S. P. Parkin, M. Hayashi, and L. Thomas, Science 320, 190 (2008).

[5] M. Bolte, G. Meier, B. Krüger, A. Drews, R. Eiselt, L. Bocklage, S. Bohlens, T. Tyliszczak, A. Vansteenkiste, B. VanWaeyenberge, K. W. Chou, A. Puzic, and H. Stoll, Phys. Rev. Lett. 100, 176601 (2008); L. Thomas, R. Moriya, C. Rettner, and S. S. P. Parkin, Science 330, 1810 (2010).

[6] L. Thomas, M. Hayashi, X. Jiang, R. Moriya, C. Rettner, and S. S. P. Parkin, Nature (London) 443, 197 (2006).

[7] S. Zhang and Z. Li, Phys. Rev. Lett. 93, 127204 (2004).

[8] A. Thiaville, Y. Nakatani, J. Miltat, and Y. Suzuki, Europhys. Lett. 69, 990 (2005).

[9] G. Tatara and H. Kohno, Phys. Rev. Lett. 92, 086601 (2004).

[10] S. Bohlens and D. Pfannkuche, Phys. Rev. Lett. 105, 177201 (2010).

[11] J. Xiao, A. Zangwill, and M. D. Stiles, Phys. Rev. B 73, 054428 (2006).

[12] G. Tatara, H. Kohno, J. Shibata, Y. Lemaho, and K-J. Lee, J. Phys. Soc. Jpn. 76, 054707 (2007).

[13] S. Zhang and Steven S.-L. Zhang, Phys. Rev. Lett. 102, 086601 (2009).

[14] I. Garate, K. Gilmore, M. D. Stiles, and A. H. MacDonald, Phys. Rev. B 79, 104416 (2009).

[15] M. Eltschka, M. Wötzel, J. Rhensius, S. Krzyk, U. Nowak, M. Kläui, T. Kasama, R. E. Dunin-Borkowski, L. J. Heyderman, H. J. van Driel, and R. A. Duine, Phys. Rev. Lett. 105, 056601 (2010).

[16] L. Heyne, J. Rhensius, D. Ilgaz, A. Bisig, U. Rüdiger, M. Kläui, L. Joly, F. Nolting, L. J. Heyderman, J. U. Thiele, and F. Kronast, Phys. Rev. Lett. 105, 187203 (2010).

[17] S. D. Pollard, L. Huang, K. S. Buchanan, D. A. Arena, and Y. Zhu, Nature Commun. 3, 1028 (2012).

[18] O. Wessely, D. M. Edwards, and J. Mathon, Phys. Rev. B 77, 174425 (2008).

[19] C. Burrowes, A. P. Mihai, D. Ravelosona, J.-V. Kim, C. Chappert, L. Vila, A. Marty, Y. Samson, F. G.-Sanchez, L. D. B.-Prejbeanu, I. Tudosa, E. E. Fullerton, and J.-P. Attané, Nature Phys. 6, 17 (2010).

[20] M. Hayashi, L. Thomas, Ya. B. Bazaliy, C. Rettner, R. Moriya, X. Jiang, and S. S. P. Parkin, Phys. Rev. Lett. 96, 197207 (2006).
[21] T. A. Moore, M. Kläui, L. Heyne, P. Möhrke, D. Backes, J. Rhensius, U. Rüdiger, L. J. Heyderman, J.-U. Thiele, G. Woltersdorf, C. H. Back, A. Fraile Rodriguez, F. Nolting, T. O. Mentes, M. A. Niño, A. Locatelli, A. Potenza, H. Marchetto, S. Cavill, and S. S. Dhesi, Phys. Rev. B. 80, 132403 (2009).

[22] O. Boulle, J. Kimling, P. Warnicke, M. Kläui, U. Rüdiger, G. Malinowski, H. J. M. Swagten, B. Koopmans, C. Ulysse, and G. Faini, Phys. Rev. Lett. 101, 216601 (2008).

[23] X. Waintal and M. Viret, Europhys. Lett. 65, 427 (2004).

[24] A. Manchon and K.-J. Lee, Appl. Phys. Lett. 99, 022504 (2011).

[25] A. Manchon, K.-S. Kim, and K.-J. Lee, arXiv:1110.3487; D. Claudio-Gonzalez, A. Thiaville, and J. Miltat, Phys. Rev. Lett. 108, 227208 (2012); A. Bisig, C. A. Akosa, J.-H. Moon, J. Rhensius, C. Moutafis, A. von Bieren, J. Heidler, G. Kiliani, M. Kammerer, M. Curcic, M. Weigand, T. Tyliszczak, B. V. Waeyenberge, H. Stoll, G. Schütz, K.-J. Lee, A. Manchon, and M. Kläui (unpublished).

[26] C. W. Groth, M. Wimmer, A. R. Akhmerov, and Xavier Waintal, New J. Phys. 16, 063065 (2014).

[27] http://www.kwant-project.org

[28] K.-J. Lee, M. D. Stiles, H.-W. Lee, J.-H. Moon, and S.-W. Lee, Phys. Rep. 531, 89 (2013).

[29] P. M. Levy and S. Zhang, Phys. Rev. Lett. 79, 5110 (1997).

[30] R. J. Elliott, Phys. Rev. 96, 266 (1954); Y. Yafet, Phys. Lett. A 98, 287 (1983).

[31] Z. Yuan, Y. Liu, A. A. Starikov, P. J. Kelly, and A. Brataas, Phys. Rev. Lett. 109, 267201 (2012).

[32] E. G. Mishchenko, A. V. Shytov, and B. I. Halperin, Phys. Rev. Lett. 93, 226602 (2004).

[33] T. Valet and A. Fert, Phys. Rev. B 48, 7099 (1993).

[34] M. D. Stiles and A. Zangwill, Phys. Rev. B 66, 014407 (2002); M. Zwierzycki, Y. Tserkovnyak, P. J. Kelly, A. Brataas, and G. E. W. Bauer, ibid. 71, 064420 (2005); S. Wang, Y. Xu, and K. Xia, ibid. 77, 184430 (2008).

[35] X. Wang and A. Manchon, Phys. Rev. Lett. 108, 117201 (2012).

[36] C. Petitjean, D. Luc, and X. Waintal, Phys. Rev. Lett. 109, 117204 (2012).

[37] Since the vectors $-\mathcal{D} \nabla^{2} \delta \mathbf{s}$ and $\delta \mathbf{s}$ are two vectors in space, they can be written in the form

$$
\begin{aligned}
-\mathcal{D} \nabla^{2} \delta \mathbf{s} & =\frac{\mathcal{D}}{W^{2}}\left(a_{1} \nabla_{x} \mathbf{m}+a_{2} \mathbf{m} \times \nabla_{x} \mathbf{m}+a_{3} \mathbf{m}\right), \\
\delta \mathbf{s} & =\frac{\mathcal{D}}{W^{2}}\left(b_{1} \nabla_{x} \mathbf{m}+b_{2} \mathbf{m} \times \nabla_{x} \mathbf{m}+b_{3} \mathbf{m}\right),
\end{aligned}
$$

where $a_{i}\left(b_{i}\right),(i=1,2,3)$ are position dependent scalars. Therefore $-\mathcal{D} \nabla^{2} \delta \mathbf{s}$ can be rewritten in the form

$$
-\mathcal{D} \nabla^{2} \delta \mathbf{s}=\frac{\mathcal{D}}{W^{2}}\left(\mathcal{G} \delta \mathbf{s}+\mathcal{B}_{a} \nabla_{x} \mathbf{m}+\mathcal{B}_{n} \mathbf{m} \times \nabla_{x} \mathbf{m}+\mathcal{B} \mathbf{m}\right),
$$

where $\mathcal{G}$ is some position dependent scalar, $\mathcal{B}_{a}=a_{1}-b_{1} \mathcal{G}$, $\mathcal{B}_{n}=a_{2}-b_{2} \mathcal{G}, \mathcal{B}=a_{3}-b_{3} \mathcal{G}$.

[38] S. Urazhdin, R. Loloee, and W. P. Pratt, Jr., Phys. Rev. B 71, 100401(R) (2005).

[39] A. A. Thiele, Phys. Rev. Lett. 30, 230 (1973). 